\title{
一种简便高效的 $\mathrm{Ta}_{2} \mathrm{O}_{5}$ 催化溴代芳烃和芳酸的 Barbier-Grignard 反应制备三级醇
}

\author{
王华然唐 渝* 杨骏张渊明*
}

(暨南大学生命科学技术学院化学系 广州 510632)

\begin{abstract}
摘要 首次报道了在四氢呋喃和甲苯的混合溶剂中, $10 \mathrm{~mol} \%$ 的 $\mathrm{Ta}_{2} \mathrm{O}_{5}$ 在 $103{ }^{\circ} \mathrm{C}$ 条件下催化不活泼溴代芳烃与芳酸进行 Barbier-Grignard 反应，“一锅法” 制备三级醇的方法. 获得了一系列具有不同取代基的三级醇，并得到了 48\% 87\%的 良好收率，同时讨论了底物空间位阻和电子效应对反应的影响，提出了 $\mathrm{Ta}_{2} \mathrm{O}_{5}$ 催化反应的可能机理.
\end{abstract}

关键词＼cjkstart芳香羧酸; Barbieri-Grignard 反应; 合成; 三级醇

\section{A Simple and Efficient Synthesis of Tertiary Alcohols via $\mathrm{Ta}_{2} \mathrm{O}_{5}-$ Catalyzed Barbier-Grignard Addition of Unactivated Aryl Bromides to Aryl Acids}

\author{
Wang, Huaran Tang, Yu* Yang, Jun Zhang, Yuanming* \\ (Department of Chemistry, Jinan University, Guangzhou 510632)
}

\begin{abstract}
Carried out in THF-toluene at $103{ }^{\circ} \mathrm{C}$, the first one-pot Barbier-Grignard reaction to synthesize tertiary alcohols has been developed and systematically investigated using aromatic acids and unactivated aryl bromides in the presence of 10 $\mathrm{mol} \%$ of $\mathrm{Ta}_{2} \mathrm{O}_{5}$. A wide range of substituted tertiary alcohols were obtained in moderate to good yields of $48 \% \sim 87 \%$. Steric and electronic effects of reactants on the reaction have been discussed. A possible mechanism catalyzed by $\mathrm{Ta}_{2} \mathrm{O}_{5}$ is proposed. Keywords aryl acid; barbier-grignard-type reaction; synthesis; tertiary alcohol
\end{abstract}

形成 $\mathrm{C}-\mathrm{C}$ 键是有机合成不可或缺的重要基础之 - ${ }^{[1]}$, 有机金属化合物与羰基化合物的反应被广泛用于 生成新的 $\mathrm{C}-\mathrm{C}$ 键 ${ }^{[2]}$, 在这些反应中, 从格氏试剂制备 各类醇是最经常使用, 也是最基本的方法 ${ }^{[3]}$. 虽然有较 多的相关研究报道, 但格氏试剂需要预先制备, 且其制 备和反应都需在严格的无水、无氧条件下进行 ${ }^{[4]}$, 这在 某些情况下限制了其使用, 因此近年来对 BarbierGrignard 反应的研究不断发展, 方兴未艾 ${ }^{[5]}$.

有关醛 ${ }^{[6]}$ 、酮 ${ }^{[6 a, 6 b, 6 e, 7]}$ 和卤代烃的 Barbier-Grignard 反应的研究相对较多, 但这些研究多局限于烯丙基、茮 基和炔丙基等活泼卤代烃 ${ }^{[8]}$. 近来有文献报道了不活泼 的碘苯和醛在水中的 Barbier-Grignard 反应 ${ }^{[9]}$, 但溴代芳 烃不能反应, 我们课题组最近报道了不活泼的溴代烃与 醛在四氢呋喃(THF)中的 Barbier-Grignard 反应, 高效、
高收率地制备了不同的加成产物醇 ${ }^{[10]}$. 另外一方面, 很 少有羧酸衍生物被用于 Barbier-Grignard 反应的研究中, 可能因为羧酸含有活泼氢，尚未见到与其相关的 Barbier-Grignard 反应的研究报道 ${ }^{[7 b, 11]} .2014$ 年我们课题 组 ${ }^{[11 \mathrm{~b}]}$ 首次报道了纳米 $\mathrm{CuO}$ 催化酯和芳基、烷基溴代物 的 Barbier-Grignard 反应，但在该条件下，芳酸酯如苯甲 酸乙酯却不能与溴苯进行 Barbier-Grignard 反应. 与羧 酸酯相比, 羧酸是一种来源更为广泛、也更为容易获得 的原料，因此寻找一种用羧酸和不活泼溴代烃有效地制 备三级醇的方法仍然是很有挑战性的课题 ${ }^{[7 b]}$.

由于含有活泼氢，羧酸很少被用于和有机金属化合 物加成合成三级醇, 这方面的研究也较少. 在早期报道 的方法中, 为了保证羧酸完全转化及提高收率, 均需加 入大过量的格氏试剂 ${ }^{[12 \sim 15]}$. 羧酸和格氏试剂加成通常

\footnotetext{
* E-mail: tytang@jnu.edu.cn, tzhangym@jnu.edu.cn

Received February 24, 2016; revised May 3, 2016; published online June 1, 2016.

Project supported by the National Natural Science Foundation of China (No. 21276104).

国家自然科学基金(No. 21276104)资助项目.
} 
只会生成少量的三级醇，同时还有部分酮，以及还原该 酮产生的二级醇等产物的生成. 如全氟取代羧酸和异丙 基溴化镁反应得到的三级醇收率非常低, 但是加成形成 的酮却达到了低到中等的收率 ${ }^{[12,13]}$, 使用镍催化剂可以 抑制反应中三级醇的生成而使酮成为主要产物 ${ }^{[14]}$. 此 外, 不加入催化剂, 虽然苯甲酸和乙烯基氯化镁可以得 到 $86 \%$ 收率的酮, 但是使用其他羧酸只能得到低到中等 收率 ${ }^{[15]}$, 这似乎说明羧酸和格氏试剂反应更倾向于生 成酮.

我们在此报道了一个只需小过量的溴代物(按反应 摩尔比, 溴代物与芳酸为 $1.5: 1) 、 10 \mathrm{~mol} \% \mathrm{Ta}_{2} \mathrm{O}_{5}$ 催化 的芳基溴代物和芳香酸在四氢呋喃和甲苯中 $103{ }^{\circ} \mathrm{C}$ 条 件下反应高效地形成三级醇的方法(图 1).

$$
\mathrm{R}^{1} \mathrm{Br}+\mathrm{R}^{2} \mathrm{COOH} \underset{\text { THF-Toluene, } 103^{\circ} \mathrm{C}}{\stackrel{\text { Mg, catalyst, addtive }}{\longrightarrow}} \mathrm{R}^{1} \underset{\mathrm{R}^{2}}{\stackrel{\mathrm{OH}}{\longrightarrow}} \mathrm{R}^{1}
$$

图 1 催化剂参与的芳香酸和芳香溴代物的 Barbier-Grignard 型反应

Scheme 1 Catalyst mediated Barbier-Grignard reaction of aryl acids with aryl bromides.

\section{1 结果与讨论}

在实验中, 苯甲酸和溴苯被用作模型化合物优化反 应条件(表 1)，基于我们对于酯的 Barbier-Grignard 反应 的研究结果 ${ }^{[11 b]}, \mathrm{CuO}$ 被首先用于催化反应, 但没有催化 效果(表 1, Entries 1,2). 我们考虑苯甲酸的酸性可能不 利于反应, 于是常见的有机碱吡定 (25 mol\%)被尝试加 入反应中，产物三苯甲醇的收率从 $45 \%$ 增加到 54\%(表 1, Entries 2, 3), 但是进一步增加吡啶用量到 $50 \mathrm{~mol} \%$, 收率基本无变化(表 1, Entry 4). 在保持 $25 \mathrm{~mol} \%$ 吡啶用 量的条件下, 催化剂 $\mathrm{NiCl}_{2} 、 \mathrm{CoCl}_{2}$ 和 $\mathrm{Pd}\left(\mathrm{CH}_{3} \mathrm{CO}_{2}\right)_{2}$ 都使 三苯甲醇的收率明显下降(表 1 , Entries 5 7). 而 $\mathrm{CuBr}$ 、 纳米 $\mathrm{CuO}$ 和 $\mathrm{Cu}_{2} \mathrm{O}$ 等其他铜催化剂催化反应获得接近 $60 \%$ 的收率(表 1, Entries 8 10), 当使用 $\mathrm{Nb}_{2} \mathrm{O}_{5}$ 和 $\mathrm{Ta}_{2} \mathrm{O}_{5}$ 作催化剂时，反应收率分别继续被提高到 $70 \%$ 和 $79 \%$ (表 1, Entries 11, 12). 以 $\mathrm{Ta}_{2} \mathrm{O}_{5}$ 作催化剂, 我们尝试 使用了 $25 \mathrm{~mol} \%$ 的其他无机或有机碱, 但反应收率都只 能维持在 $70 \%$ 左右(表 1, Entries 13 16), 因此 $\mathrm{Ta}_{2} \mathrm{O}_{5}$ 和 $25 \mathrm{~mol} \%$ 的吡啶被用于下面的讨论中. 在没有催化剂, 只添加 $25 \mathrm{~mol} \%$ 吡啶的反应中, 获得 $61 \%$ 收率的三苯甲 醇(表 1, Entry 17), 比没有催化剂也没有吡啶的反应结 果明显要高(表 1, Entry 1), 这表明吡啶是通过与苯甲酸 作用而促进反应.

表 2 列出了温度、催化剂用量对反应收率的影响. 单独用 $\mathrm{THF}$ 作为溶剂, 在 $65{ }^{\circ} \mathrm{C}$ 回流条件下反应, 产物
表 1 碱和催化剂对反应影响 ${ }^{a}$

Table 1 Effects of bases and catalysts on the reaction

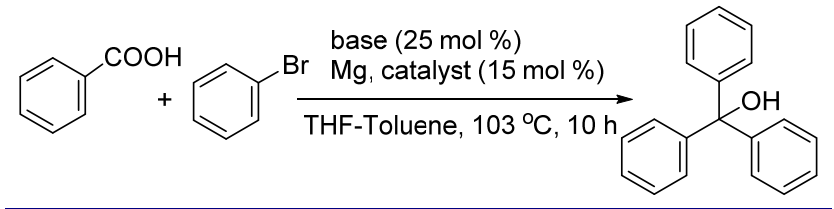

\begin{tabular}{cllc}
\hline Entry & Catalyst & \multicolumn{1}{c}{ Base } & Yield $^{b} \%$ \\
\hline 1 & $\mathrm{None}$ & None & 43 \\
2 & $\mathrm{CuO}$ & None & 45 \\
3 & $\mathrm{CuO}$ & Pyridine & 54 \\
$4^{c}$ & $\mathrm{CuO}$ & Pyridine & 52 \\
5 & $\mathrm{NiCl}_{2}$ & Pyridine & 31 \\
6 & $\mathrm{CoCl}_{2}$ & Pyridine & Trace \\
7 & $\mathrm{Pd}_{2}\left(\mathrm{CH}_{3} \mathrm{COO}\right)_{2}$ & Pyridine & 6 \\
8 & $\mathrm{CuBr}$ & Pyridine & 62 \\
9 & $\mathrm{CuO}($ nano $)$ & Pyridine & 60 \\
10 & $\mathrm{Cu}_{2} \mathrm{O}$ & Pyridine & 59 \\
11 & $\mathrm{Nb}_{2} \mathrm{O}_{5}$ & Pyridine & 70 \\
12 & $\mathrm{Ta}_{2} \mathrm{O}_{5}$ & Pyridine & 79 \\
13 & $\mathrm{Ta}_{2} \mathrm{O}_{5}$ & KOH & 67 \\
14 & $\mathrm{Ta}_{2} \mathrm{O}_{5}$ & Triethylamine & 69 \\
15 & $\mathrm{Ta}_{2} \mathrm{O}_{5}$ & $N$-Methylmorpholine & 71 \\
16 & $\mathrm{Ta}_{2} \mathrm{O}_{5}$ & $N, N$-Dimethylcyclohexylamine & 69 \\
17 & $\mathrm{None}$ & Pyridine & 61 \\
\hline
\end{tabular}

${ }^{a}$ Reaction conditions: $\mathrm{PhBr}$ (15 mmol, 3 equiv.), $\mathrm{Mg}$ (15 mmol, 3 equiv.), benzoic acid ( $5 \mathrm{mmol}, 1$ equiv.), base $(25 \mathrm{~mol} \%)$, catalyst $(15 \mathrm{~mol} \%$ ) reacting in $5 \mathrm{~mL}$ of THF and $20 \mathrm{~mL}$ of toluene for $10 \mathrm{~h}^{b}{ }^{b}$ Isolated yield. ${ }^{c} 50 \mathrm{~mol} \%$ of pyridine.

表 2 温度和催化剂用量对反应的影响 ${ }^{a}$

Table 2 Effects of temperature and catalyst amount on the reaction

\begin{tabular}{|c|c|c|c|}
\hline & & $\begin{array}{l}\text { pyridine }(25 \mathrm{~mol} \%) \\
\mathrm{Mg}, \mathrm{Ta}_{2} \mathrm{O}_{5}(\mathrm{X} \mathrm{mol} \%)\end{array}$ & \\
\hline Entry & $T /{ }^{\circ} \mathrm{C}$ & Catalyst $/ \mathrm{mol} \%$ & Yield $^{b} / \%$ \\
\hline $1^{c}$ & 65 & 15 & 43 \\
\hline $2^{d}$ & 78 & 15 & 67 \\
\hline 3 & 103 & 15 & 79 \\
\hline 4 & 103 & 25 & 37 \\
\hline 5 & 103 & 10 & 84 \\
\hline 6 & 103 & 5 & 70 \\
\hline
\end{tabular}

${ }^{a}$ Reaction conditions: $\mathrm{PhBr}$ (15 mmol, 3 equiv.), $\mathrm{Mg}(15 \mathrm{mmol}, 3$ equiv.), benzoic acid ( $5 \mathrm{mmol}, 1$ equiv.), pyridine ( $25 \mathrm{~mol} \%$ ), reacting in $5 \mathrm{~mL}$ of THF and $20 \mathrm{~mL}$ of toluene at $103{ }^{\circ} \mathrm{C}$ for $10 \mathrm{~h} .{ }^{b}$ Isolated yield. ${ }^{c}$ Reacting in $25 \mathrm{~mL}$ THF. ${ }^{d}$ Reacting in $15 \mathrm{~mL}$ THF and $10 \mathrm{~mL}$ toluene.

收率为 $43 \%$ (表 2, Entry 1). 在 THF 中加入甲苯分别提升 反应温度至 $78{ }^{\circ} \mathrm{C}(10 \mathrm{~mL} \mathrm{THF}+15 \mathrm{~mL}$ 甲苯 $)$ 和 $103{ }^{\circ} \mathrm{C}$ $(5 \mathrm{~mL} \mathrm{THF}+20 \mathrm{~mL}$ 甲苯)时, 反应收率分别为 $67 \%$ 和 $79 \%$ (表 2, Entries 2, 3), 可以看到温度升高对反应有利.

进一步增加溶剂中甲苯比例, 虽然能略微提高温 度, 但是由于 THF 的量太少, 不利于溴苯与镁的反应, 
因此收率反而明显降低. 催化剂用量增加到 $25 \mathrm{~mol} \%$ 时, 反应收率显著下降到 37\%(表 2, Entry 4); 催化剂用量从 $15 \mathrm{~mol} \%$ 降至 $10 \mathrm{~mol} \%$ 时, 收率从 79\%(表 2, Entry 3)略 提升到 84\%(表 2, Entry 5), $15 \mathrm{~mol} \% \mathrm{Ta}_{2} \mathrm{O}_{5}$ 催化时, 二苯 甲酮的分离收率约 $15 \%$, 高于 $10 \mathrm{~mol} \% \mathrm{Ta}_{2} \mathrm{O}_{5}$ 催化时的 $7 \%$. 因此催化剂用量的增加, 有可能会因促进副产物 的增加而导致三苯甲醇收率降低. 继续减少催化剂用量 到 $5 \mathrm{~mol} \%$, 反应收率降低到 70\%(表 2, Entry 6), 表明 10 $\mathrm{mol} \%$ 的催化剂用量较合适.

随后我们探讨了反应时间、镁和溴苯用量以及加入 配体对反应的影响. 反应从 $1.5 \mathrm{~h}$ 延长至 $3 \mathrm{~h}$, 收率从 65\%提高到 87\%(表 3, Entries 1, 2), 但进一步延长反应 时间时, 收率呈现下降趋势(表 3, Entries 3 5). 溴苯和 镁用量从 3 倍量增加到 $3.5 \sim 4$ 倍量时, 反应收率变化不 大(表 3, Entries 6,7), 但是减少溴苯和镁用量到 2.5 和 2 倍，反应收率却明显下降，为 55\%和 31\%(表 3, Entries 8 , 9).

表 3 反应时间、溴苯和镁的用量以及配体对反应影响 ${ }^{a}$

Table 3 Effects of reaction time, amount of $\mathrm{Mg}$ and $\mathrm{PhBr}$, and different ligands on the reaction

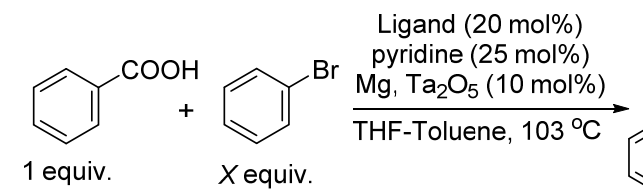

\begin{tabular}{cclcc}
\hline Entry & $X$ /equiv. & Ligand & Time $/ \mathrm{h}$ & Yield $^{b} / \%$ \\
\hline 1 & 3.0 & None & 1.5 & 65 \\
2 & 3.0 & None & 3 & 87 \\
3 & 3.0 & None & 4.5 & 78 \\
4 & 3.0 & None & 6 & 75 \\
5 & 3.0 & None & 8 & 73 \\
6 & 3.5 & None & 3 & 87 \\
7 & 4.0 & None & 3 & 88 \\
8 & 2.5 & None & 3 & 55 \\
9 & 2.0 & None & 3 & 31 \\
10 & 6.0 & None & 3 & 86 \\
11 & 3.0 & PPh & 3 & 67 \\
12 & 3.0 & DPPP & 3 & 68 \\
13 & 3.0 & $L_{-C y s t e i n e}$ & 3 & 48 \\
14 & 3.0 & TED & 3 & 55 \\
15 & 3.0 & Glycine & 3 & 49 \\
16 & 3.0 & PMDETA & 3 & 54 \\
17 & 3.0 & BDMAEE & 3 & 57 \\
\hline
\end{tabular}

${ }^{a}$ Reaction conditions: $\mathrm{PhBr}(X$ equiv.), $\mathrm{Mg}$ ( $X$ equiv.), benzoic acid $(5 \mathrm{mmol}$, 1equiv), $\mathrm{Ta}_{2} \mathrm{O}_{5}$, pyridine and ligand, reacting in $5 \mathrm{~mL}$ of THF and $20 \mathrm{~mL}$ of toluene. ${ }^{b}$ Isolated yield. ${ }^{c} \mathrm{Bis}$ (2-dimethylaminoethyl) ether. ${ }^{d}$ No pyridine or ligand.

不添加吡啶, $\mathrm{Ta}_{2} \mathrm{O}_{5}$ 催化 6 倍量溴苯和苯甲酸反应的 收率为 $86 \%$, 与添加 $25 \mathrm{~mol} \%$ 吡啶, 使用 3 倍量溴苯的 收率相近(表 3, Entries 2,10), 可以看到, 加入 $25 \mathrm{~mol} \%$
吡啶可以减少 3 倍溴苯的用量，体现出了吡啶在此反应 的重要作用。一些常见的氮、磷配体对反应没有促进作 用，反而使反应收率降到了 $48 \% \sim 68 \%$ (表 3, Entries $11 \sim 17)$, 因此最后确定较优反应条件为: 使用苯甲酸 3 倍量的溴苯和镁、 $10 \mathrm{~mol} \%$ 的 $\mathrm{Ta}_{2} \mathrm{O}_{5}$ 和 $25 \mathrm{~mol} \%$ 吡啶在 5 $\mathrm{mL} \mathrm{THF}$ 和 $20 \mathrm{~mL}$ 甲苯混合溶剂的共沸温度即 $103{ }^{\circ} \mathrm{C}$ 条 件下反应 $3 \mathrm{~h}$. 此外, 由于 THF 沸点低易挥发同时在混 合溶剂中比例较少, 我们在冷凝管上端套上一个气球, 密封三口瓶的玻璃塞则用生料带缠紧, 减少 THF 挥发 对反应的影响 ${ }^{[7 \mathrm{~d}]}$.

采用上述较优反应条件, 我们考察了反应对于不同 的溴代物和芳酸的耐受性(表 4). 在与苯甲酸的反应中, 具有供电子基团的对甲氧基溴苯和对甲基溴苯都能获 得超过 $80 \%$ 的较好收率，与溴苯的反应结果接近(表 4 , Entries 1 3). 从邻甲基溴苯和邻甲氧基溴得到的三级 醇产物的收率分别只有 $61 \%$ 和 53\%, 表现出明显的空间 位阻效应影响(表 4, Entries 4, 5). 弱的吸电子作用使间 甲氧基溴苯生成收率 71\%的产物(表 4, Entry 6), 虽比其 对位同系物所获收率低，但仍比其邻位同系物收率高， 仍然相对较好. 与间氟溴苯、对氯溴苯对应的反应收率

表 4 较优反应条件下合成不同结构三级醇的收率 ${ }^{a}$

Table 4 Screening of the optimum reacting conditions for synthesis of tertiary alcohol compounds

$$
\mathrm{R}^{1} \mathrm{Br}+\mathrm{R}^{2} \mathrm{COOH} \underset{\mathrm{THF}-\text { Toluene } 103^{\circ} \mathrm{C}}{\stackrel{\mathrm{Mg}_{1} \mathrm{Ta}_{2} \mathrm{O}_{5}(10 \mathrm{~mol} \%)}{\longrightarrow}} \mathrm{R}^{1} \underset{\mathrm{OH}}{\mathrm{R}^{2}} \mathrm{R}^{1}
$$

\begin{tabular}{|c|c|c|c|c|c|}
\hline \multirow{2}{*}{$\frac{\text { Entry }}{1}$} & \multirow{2}{*}{$\frac{\mathrm{R}^{1}}{\mathrm{Ph}}$} & \multirow{2}{*}{$\frac{\mathrm{R}^{2}}{\mathrm{C}_{6} \mathrm{H}_{5}}$} & \multicolumn{2}{|c|}{ Product Time ${ }^{b} / \mathrm{h}$} & \multirow{2}{*}{$\frac{\text { Yield }^{c} / \%}{87}$} \\
\hline & & & $4 a$ & 3 & \\
\hline 2 & $4-\mathrm{MeC}_{6} \mathrm{H}_{4}$ & $\mathrm{C}_{6} \mathrm{H}_{5}$ & $4 b$ & 3 & 85 \\
\hline 3 & 4- $\mathrm{MeOC}_{6} \mathrm{H}_{4}$ & $\mathrm{C}_{6} \mathrm{H}_{5}$ & $4 c$ & 5 & 81 \\
\hline 4 & 2-Me $\mathrm{C}_{6} \mathrm{H}_{4}$ & $\mathrm{C}_{6} \mathrm{H}_{5}$ & 4d & 4 & 61 \\
\hline 5 & $2-\mathrm{MeOC}_{6} \mathrm{H}_{4}$ & $\mathrm{C}_{6} \mathrm{H}_{5}$ & $4 e$ & 5 & 53 \\
\hline 6 & $3-\mathrm{MeOC}_{6} \mathrm{H}_{4}$ & $\mathrm{C}_{6} \mathrm{H}_{5}$ & $4 f$ & 3 & 71 \\
\hline 7 & $3-\mathrm{FC}_{6} \mathrm{H}_{4}$ & $\mathrm{C}_{6} \mathrm{H}_{5}$ & $4 g$ & 5 & 72 \\
\hline 8 & 4- $\mathrm{ClC}_{6} \mathrm{H}_{4}$ & $\mathrm{C}_{6} \mathrm{H}_{5}$ & $4 h$ & 4 & 48 \\
\hline 9 & 1-Naphthyl & $\mathrm{C}_{6} \mathrm{H}_{5}$ & $4 i$ & 10 & 57 \\
\hline 10 & 2-Thienyl & $\mathrm{C}_{6} \mathrm{H}_{5}$ & $4 j$ & 3 & 84 \\
\hline 11 & Cyclohexyl & $\mathrm{C}_{6} \mathrm{H}_{5}$ & $4 k$ & 3 & $12\left(36^{d}\right)$ \\
\hline 12 & $\mathrm{Ph}$ & $4-\mathrm{MeC}_{6} \mathrm{H}_{4}$ & 41 & 3 & 77 \\
\hline 13 & $\mathrm{Ph}$ & 4-t-ButylC ${ }_{6} \mathrm{H}_{4}$ & $4 m$ & 4 & 63 \\
\hline 14 & $4-\mathrm{MeOC}_{6} \mathrm{H}_{4}$ & 4-t-ButylC ${ }_{6} \mathrm{H}_{4}$ & $4 n^{e}$ & 3 & 68 \\
\hline 15 & $\mathrm{Ph}$ & $2-\mathrm{ClC}_{6} \mathrm{H}_{4}$ & 40 & 10 & 48 \\
\hline 16 & 2- $\mathrm{MeOC}_{6} \mathrm{H}_{4}$ & 4- $t$-ButylC ${ }_{6} \mathrm{H}_{4}$ & $4 p^{e}$ & 3 & 50 \\
\hline 17 & 2- $\mathrm{MeOC}_{6} \mathrm{H}_{4}$ & $4-\mathrm{MeC}_{6} \mathrm{H}_{4}$ & $\mathbf{4 q} \mathbf{q}^{e}$ & 5 & 63 \\
\hline 18 & $2-\mathrm{MeC}_{6} \mathrm{H}_{4}$ & $4-\mathrm{MeC}_{6} \mathrm{H}_{4}$ & $4 r^{e}$ & 3 & 58 \\
\hline 19 & $\mathrm{Ph}$ & $i$-Bu & $4 s$ & 10 & 56 \\
\hline
\end{tabular}

${ }^{a}$ Reaction conditions: $\mathrm{PhBr}$ (15 mmol, 3 equiv.), $\mathrm{Mg}$ (15 mmol, 3 equiv.), benzoic acid ( $5 \mathrm{mmol}, 1$ equiv.), $\mathrm{Ta}_{2} \mathrm{O}_{5}$ and pyridine, reacting in $5 \mathrm{~mL}$ of THF and $20 \mathrm{~mL}$ of toluene. ${ }^{b}$ Determined by TLC ${ }^{c}$ Isolated yield. ${ }^{d} 7$ equiv. of cyclohexyl bromide and magnesium employed. ${ }^{e}$ New compound. 
分别为 72\%和 48\%(表 4, Entries 7,8), 间氟取代时的吸 电子效应对反应收率的影响与间甲氧基接近, 对氯溴苯 可能存在别的作用，其对应的反应收率明显下降.

1-溴代萗的反应活性相对比较低, 因此反应时间较 长, 获得了中等的反应收率 57\%(表 4, Entry 9), 2-溴噻吩 表现出了与溴苯接近的反应活性, 获得了 $84 \%$ 的较好收 率(表 4, Entry 10). 需要说明的是, 上面这些反应中, 通 过 TLC 检测苯甲酸都已完全转化, 与三级醇同时产生 的副产物二级醇和酮是导致目标产物收率下降的一个 重要原因. 当用环已基溴与苯甲酸的反应时, 3 倍量的 环己基溴得到的三级醇的收率为 12\%(表 4, Entry 11), 由于烷基溴代物的反应活性比芳基溴代物低 ${ }^{[1 b]}$, 因此 环已基溴和镁的用量被增加到 7 倍，但对应的三级醇的 收率虽明显增加到 36\%, 但也仍然较低(表 4, Entry 11).

虽然比从苯甲酸制备三级醇的收率低, 烷基取代的 苯甲酸也得到中等偏好收率 $(63 \% \sim 77 \%)$ 的三级醇产物 (表 4, Entries 12 14). 邻氯苯甲酸和溴苯反应收率得到 48\%的收率(表 4, Entry 15). 2-甲氧基溴苯和 2-甲基溴苯 只能获得 50\% 63\%中等收率的产物(表 4, Entries 16 18). 澳苯和异丁酸反应得到 56\%收率的三级醇(表 4, Entry 19), 但是其他脂肪族酸如棕榈酸, 与溴苯反应 20 $\mathrm{h}$ 只能得到约 $20 \%$ 的三级醇, 而乙酸即使与溴苯反应 24 $\mathrm{h}$ 也没有检测到目标产物的生成.

根据文献报道, 无催化剂时, 苯甲酸与格氏试剂反 应的主要产物为三级醇, 加入催化剂 $\mathrm{Ni}(\mathrm{DPPE}) \mathrm{Cl}_{2}$, 则 主要产物为酩 ${ }^{[14]}$, 但需用 6 倍量的格氏试剂, 羧酸才能 完全转化, 使用 3 倍量格氏试剂反应时, 有 $50 \%$ 的羧酸 被回收. 为了更好地理解本文所讨论的反应, 在其他最 优条件保持不变、 $\mathrm{Ni}$ (DPPE) $\mathrm{Cl}_{2}$ 催化的情况下, 6 倍量的 溴苯和镁被用于与苯甲酸反应, 得到了 $30 \%$ 的二苯甲酮 以及少量的三苯甲醇. 这一结果与 $\mathrm{Ni}(\mathrm{DPPE}) \mathrm{Cl}_{2}$ 催化的 苯甲酸与格氏试剂反应的趋势一致, 但酮的收率却比较 低，也表明 Barbier-Grignard 反应更加适合用于制备三 芳基醇而不是酮.

在反应开始发生 $5 \mathrm{~min}$ 后, 我们用 TLC 进行监控发 现三苯甲醇和二苯甲酮同时出现, 从斑点的大小初步判 断三苯甲醇相对较多. 当我们在较优反应条件下, 用 1.1 倍量的 $\mathrm{Mg}$ 和溴苯与苯甲酸连续反应 $12 \mathrm{~h}$, 得到二苯 甲酮和三苯甲醇收率分别为 $9 \%$ 和 $21 \%$, 这与我们在 3 倍量溴苯与苯甲酸反应初期, 从 TLC 监测结果的初步 判断一致, 如果溴苯与苯甲酸的加成反应先生成二苯甲 酮, 然后其再与溴苯反应得到三苯甲醇, 在上面的反应 中应该得到二苯甲酮比三苯甲醇多的结果, 而事实并非 如此. 因此我们认为三苯甲醇可能是直接由溴苯与苯甲 酸生成, 而不是经二苯甲酮转化得来. 此外, 以二苯甲
酮代替苯甲酸和 3 倍量的溴苯、镁反应 $3 \mathrm{~h}$ 得到的三苯 甲醇收率为 $69 \%$, 并回收到 $16 \%$ 的二苯甲酮，需要延长 反应时间到 $10 \mathrm{~h}$ 三苯甲醇的收率才能达到 $82 \%$, 这说明 溴苯与二苯甲酩反应的速度比与苯甲酸的反应低. 这进 一步证明我们关于三苯甲醇来源的观点, 也与我们获得 的 $\mathrm{CuO}$ 催化溴苯与乙酸乙酯的 Barbier-Grignard 反应机 理相似 ${ }^{[11 b]}$. 但两者使用的催化剂不同, 催化剂的效果 也不同, $\mathrm{CuO}$ 不能催化溴苯与酮的反应，而 $\mathrm{Ta}_{2} \mathrm{O}_{5}$ 可以. 钽具有 $5 \mathrm{~d}$ 空轨道, 而且钽酸 $\left(\mathrm{Ta}_{2} \mathrm{O}_{5} \bullet n \mathrm{H}_{2} \mathrm{O}\right)$ 具有和铌酸 $\left(\mathrm{Nb}_{2} \mathrm{O}_{5} \bullet n \mathrm{H}_{2} \mathrm{O}\right)$ 相近的空间构型 ${ }^{[16]}, \mathrm{Ta}_{2} \mathrm{O}_{5}$ 的结构有利于 其与氧原子络合. 结合上述讨论, 我们提出了一个 $\mathrm{Ta}_{2} \mathrm{O}_{5}$ 催化澳代芳烃与苯甲酸进行 Grignard-Barbier 反应 的可能机理(Scheme 2).

首先, 芳香酸和吡啶生成盐 $\mathbf{1}$, 减弱了酸的酸性, 这有利于溴苯与 $\mathrm{Mg}$ 反应, 然后 $\mathrm{Ta}_{2} \mathrm{O}_{5}$ 与羒酸中的氧原 子结合形成络合物 2 , 这有利于断开碳氧键, 接下来现 场生成的 $\mathrm{RMgBr}$ 与络合物 $\mathbf{2}$ 的羰基加成得到中间体 $\mathbf{3}$, 由于中间体 3 存在一个碳原子与两个氧原子成键的结构 而不稳定, 其被另一分子 $\mathrm{RMgBr}$ 进攻时, 在吡啶和 $\mathrm{Ta}_{2} \mathrm{O}_{5}$ 的辅助下, 中间体 3 断开一个碳氧键形成三级醇, 并释放出吡啶、 $\mathrm{Ta}_{2} \mathrm{O}_{5}$ 在反应中循环. 可能 $\mathrm{Ta}_{2} \mathrm{O}_{5}$ 通过与 二苯甲酮的嶡基氧作用, 使其能催化溴苯与二苯甲酮的 反应，而在中间体 2 和 3 中， $\mathrm{Ta}_{2} \mathrm{O}_{5}$ 与羧酸形成的络合物 具有六元环结构, 相对稳定, 因此 $\mathrm{Ta}_{2} \mathrm{O}_{5}$ 催化溴苯与苯 甲酸的反应比与二苯甲酮反应的速度快, 进一步的理解 则需要更多的实验结果支撑, 将在今后的研究中进行, 并可以考虑中间体 2 或 3 在其他反应中的应用.

\section{2 结论}

成功探索出了一种简单、有效的 $\mathrm{Ta}_{2} \mathrm{O}_{5}$ 催化的芳酸 和不活泼芳基溴代物, 在 THF 和甲苯混合溶剂中 $103{ }^{\circ} \mathrm{C}$ 条件下进行 Barbier-Grignard 反应制备三级醇的 方法，在较优反应条件下获得中等到良好的反应收率， 提出了 $\mathrm{Ta}_{2} \mathrm{O}_{5}$ 催化溴代物与羧酸反应直接生成三级醇的 合理反应机理, 这也是关于芳香酸和不活泼溴代物进行 Barbier-Grignard 反应的首次报道.

\section{3 实验部分}

\section{1 仪器与试剂}

核磁共振谱 Bruker $300 \mathrm{M}$ 核磁共振仪测定, 以 $\mathrm{CDCl}_{3}$ 作溶剂, 质谱用 Finnigan Trace GC/MS 仪测定, 红外光谱Eqinox 55 FT-IR 红外光谱仪测定, 实验所用试 剂均为市售化学纯或分析纯试剂.

\section{2 实验方法}

在 $50 \mathrm{~mL}$ 的三口烧瓶中加入 $15 \mathrm{mmol}$ 镁屑(打磨掉 


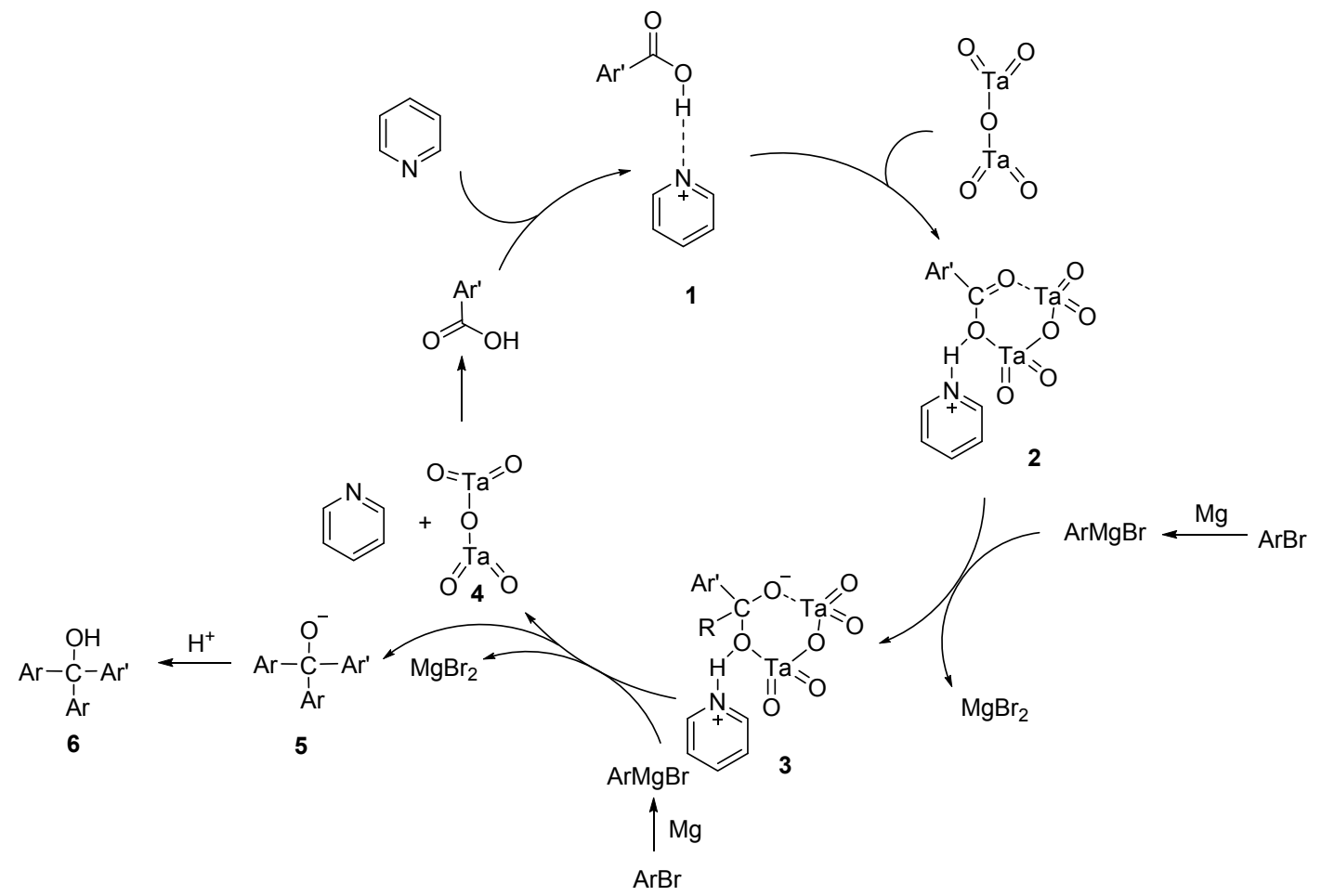

图 2 可能的反应历程

Scheme 2 Proposed mechanism

镁条表面的氧化膜, 然后剪碎), $5 \mathrm{~mL}$ 的未经处理的分 析纯 $\mathrm{THF}$ 和 $20 \mathrm{~mL}$ 甲苯, $5 \mathrm{mmol}$ 苯甲酸, $15 \mathrm{mmol}$ 溴 苯, $25 \mathrm{~mol} \%$ 吡啶, $10 \mathrm{~mol} \%$ 催化剂 $\mathrm{Ta}_{2} \mathrm{O}_{5}$, 连接好冷凝管, 并在冷凝管上端用气球密封, 三口瓶的玻璃塞用生料带 密封, 以减少因 THF 挥发带来的对反应的影响 ${ }^{[7 \mathrm{~d}]}$, 摚拌 下将混合物加热到 $103{ }^{\circ} \mathrm{C}$ 恒温回流反应一定时间, 然 后冷却反应液到室温, 加入用 $15 \mathrm{~mL}$ 饱和氯化铵溶液摚 拌淬灭反应, 抽滤反应液, 用少量乙酸乙酯洗涤滤渣, 滤液用乙酸乙酯萃取 $(3 \times 10 \mathrm{~mL})$, 合并有机层, 将有机 层用无水硫酸镁干燥、过滤、旋转蒸发得到粗产物. 称 重, 并用薄层层析法(TLC)进行分离提纯得到分离收率 [所用展开剂为 $V$ (石油醚) : $V$ (乙酸乙酯 $)=8: 1$ ]. 目标 化合物的结构经 ${ }^{1} \mathrm{H}$ NMR, IR, MS 确证并和文献对比, 新化合物通过 ${ }^{13} \mathrm{C}$ NMR 和 HRMS 进一步证实.

三苯基甲醇 (4a) ${ }^{[17]}$ : 白色固体, m.p. $163.8 \sim$ $164.4{ }^{\circ} \mathrm{C}$ (文献值 m.p. $160 \sim 163{ }^{\circ} \mathrm{C}$ ); ${ }^{1} \mathrm{H}$ NMR $(300$ $\left.\mathrm{MHz}, \mathrm{CDCl}_{3}\right) \delta: 7.46 \sim 7.36(\mathrm{~m}, 15 \mathrm{H}), 3.06(\mathrm{~s}, 1 \mathrm{H})$; IR (KBr) $v$ : 3466, 3059, 1489, 1445, 1327, 1154, 1008, 890, 759, $637 \mathrm{~cm}^{-1}$; MS (EI) m/z: $260\left(\mathrm{M}^{+}\right), 183,154,105,77$.

1,1-二(4-甲基苯基)-1-苯基甲醇(4b) ${ }^{[18]}$ : 白色固体, m.p. $75 \sim 77{ }^{\circ} \mathrm{C}$ (文献值 m.p. $70 \sim 71{ }^{\circ} \mathrm{C}$ ); ${ }^{1} \mathrm{H}$ NMR $(300$ $\left.\mathrm{MHz}, \mathrm{CDCl}_{3}\right) \delta: 7.32$ (s, $\left.5 \mathrm{H}\right), 7.17$ (d, $\left.J=5.8 \mathrm{~Hz}, 8 \mathrm{H}\right)$, 2.78 (s, 1H), 2.38 (s, 6H); IR (KBr) v: 3471, 3056, 2918, 1508, 1445, 1152, 1008, 814, 752, 723, $602 \mathrm{~cm}^{-1}$; MS (EI) $m / z: 288\left(\mathrm{M}^{+}\right), 211,119,105,91,77$.

1,1-二(4-甲氧基苯基)-1-苯基甲醇 (4c) ${ }^{[19]}$ : 白色固 体, m.p. $76 \sim 79{ }^{\circ} \mathrm{C}$ (文献值 m.p. $77 \sim 80{ }^{\circ} \mathrm{C}$ ); ${ }^{1} \mathrm{H}$ NMR $\left(300 \mathrm{MHz}, \mathrm{CDCl}_{3}\right) \delta: 7.74 \sim 6.44(\mathrm{~m}, 14 \mathrm{H}), 3.83(\mathrm{~s}, 6 \mathrm{H})$; IR (KBr) v: 3493, 2954, 2835, 1608, 1509, 1447, 1248, 1177, 1003, 830, 729, $584 \mathrm{~cm}^{-1}$; MS (EI) $m / z: 320\left(\mathrm{M}^{+}\right)$, $303,243,135,105,77$.

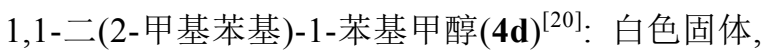
m.p. $81 \sim 82{ }^{\circ} \mathrm{C}$ (文献值 m.p. $81 \sim 83{ }^{\circ} \mathrm{C}$ ); ${ }^{1} \mathrm{H}$ NMR $(300$ $\left.\mathrm{MHz}, \mathrm{CDCl}_{3}\right) \delta: 7.35(\mathrm{t}, J=6.1 \mathrm{~Hz}, 4 \mathrm{H}), 7.28 \sim 6.66(\mathrm{~m}$, 9H), 3.01 (s, 1H), 2.24 (s, 6H); IR (KBr) v: 3557, 3058, 1658, 1599, 1486, 1448, 1006, 830, 759, $640 \mathrm{~cm}^{-1}$; MS (EI) $m / z: 20\left(\mathrm{M}^{+}\right), 270,211,197,119,105,91$. 1,1-二-(2-甲氧基苯基)-1-苯基甲醇 $(4 \mathbf{e})^{[21]}$ : 白色固 体, m.p. $112 \sim 113{ }^{\circ} \mathrm{C}$ (文献值 m.p. $115.8{ }^{\circ} \mathrm{C}$ ); ${ }^{1} \mathrm{H}$ NMR $\left(300 \mathrm{MHz}, \mathrm{CDCl}_{3}\right) \delta: 7.35 \sim 7.25(\mathrm{~m}, 7 \mathrm{H}), 7.01$ (dd, $J=$ $7.7,1.8 \mathrm{~Hz}, 2 \mathrm{H}), 6.95$ (d, $J=8.8 \mathrm{~Hz}, 3 \mathrm{H}), 6.89$ (d, $J=7.4$ $\mathrm{Hz}, 2 \mathrm{H}), 3.56$ (s, 6H); IR (KBr) v: 3495, 3002, 2834, 1591, 1034, $779 \mathrm{~cm}^{-1}$; MS (EI) m/z: $320\left(\mathrm{M}^{+}\right), 303,243,213$, $185,135,105,77$.

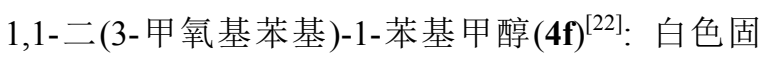
体, m.p. 77 80 ${ }^{\circ} \mathrm{C}$ (文献值 m.p. 82 $83{ }^{\circ} \mathrm{C}$ ); ${ }^{1} \mathrm{H}$ NMR $\left(300 \mathrm{MHz}, \mathrm{CDCl}_{3}\right) \delta: 7.36 \sim 7.20(\mathrm{~m}, 7 \mathrm{H}), 6.94 \sim 6.90(\mathrm{~m}$, 2H), $6.89 \sim 6.81(\mathrm{~m}, 5 \mathrm{H}), 3.77$ (s, 6H); IR (KBr) $v: 3495$, 
3002, 2834, 1591, 1034, $779 \mathrm{~cm}^{-1}$; MS (EI) m/z: 320 $\left(\mathrm{M}^{+}\right), 303,243,213,185,135,105,77$.

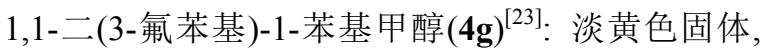
m.p. $114 \sim 116{ }^{\circ} \mathrm{C}$ (文献值 m.p. $113 \sim 114{ }^{\circ} \mathrm{C}$ ); ${ }^{1} \mathrm{H}$ NMR $\left(300 \mathrm{MHz}, \mathrm{CDCl}_{3}\right) \delta: 7.68 \sim 7.27(\mathrm{~m}, 7 \mathrm{H}), 7.24 \sim 6.94(\mathrm{~m}$, $6 \mathrm{H}), 2.85$ (s, 1H); IR (KBr) v: 2928, 2362, 1568, 1470, 1292, 1196, 1031, $856 \mathrm{~cm}^{-1}$; MS(EI) m/z: $296\left(\mathrm{M}^{+}\right), 219$, 201, 123, 105, 95, 77.

1,1-二(4-氯苯基)-1-苯基甲醇 (4h $)^{[24]}$ : 淡黄色固体, m.p. $86 \sim 87{ }^{\circ} \mathrm{C}$ (文献值 m.p. $87 \sim 88{ }^{\circ} \mathrm{C}$ ); ${ }^{1} \mathrm{H}$ NMR $(300$ $\left.\mathrm{MHz}, \mathrm{CDCl}_{3}\right) \delta: 7.40 \sim 7.29(\mathrm{~m}, 10 \mathrm{H}), 7.28 \sim 7.20(\mathrm{~m}$, $3 \mathrm{H}), 1.52 \sim 1.01(\mathrm{~m}, 1 \mathrm{H})$. IR (KBr) $v: 3454,3062,2822$, 1488, 1093, 1011, 823, 783, $701 \mathrm{~cm}^{-1}$; MS (EI) $\mathrm{m} / z: 328$ $\left(\mathrm{M}^{+}\right), 251,216,138,105,77$.

1,1-二(1-萗基)-1-苯基甲醇 $(\mathbf{4 i})^{[25]}$ : 白色固体，m.p. $167 \sim 170{ }^{\circ} \mathrm{C}$ (文献值 m.p. $165{ }^{\circ} \mathrm{C}$ ); ${ }^{1} \mathrm{H}$ NMR $(300 \mathrm{MHz}$, $\left.\mathrm{CDCl}_{3}\right) \delta: 8.35(\mathrm{~d}, J=8.7 \mathrm{~Hz}, 2 \mathrm{H}), 7.93(\mathrm{~d}, J=8.2 \mathrm{~Hz}$, 2H), 7.86 (d, $J=8.2 \mathrm{~Hz}, 2 \mathrm{H}), 7.49$ (s, 1H), 7.45 (d, $J=7.8$ $\mathrm{Hz}, 3 \mathrm{H}), 7.37$ (d, $J=7.5 \mathrm{~Hz}, 3 \mathrm{H}), 7.33$ (s, 1H), 7.27 (s, $1 \mathrm{H}), 7.24(\mathrm{~s}, 1 \mathrm{H}), 6.93(\mathrm{~d}, J=7.3 \mathrm{~Hz}, 2 \mathrm{H}), 3.72(\mathrm{~s}, 1 \mathrm{H}$, $\mathrm{OH})$; IR (KBr) $v: 3548,3048,1597,1501,1341,996,779$ $\mathrm{cm}^{-1}$; MS (EI) $m / z: 360\left(\mathrm{M}^{+}\right), 303,283,255,128,105$, 102,77 .

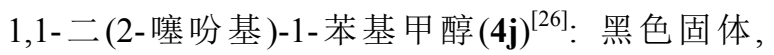
m.p. 92 93 ${ }^{\circ} \mathrm{C}$ (文献值 m.p. $\left.90 \sim 91{ }^{\circ} \mathrm{C}\right) ;{ }^{1} \mathrm{H}$ NMR (300 $\left.\mathrm{MHz}, \mathrm{CDCl}_{3}\right) \delta: 7.74 \sim 7.30(\mathrm{~m}, 7 \mathrm{H}), 7.17 \sim 6.65(\mathrm{~m}, 4 \mathrm{H})$, 3.17 (s, 1H); IR (KBr) v: 3055, 2369, 1468, 1285, 1025, $698 \mathrm{~cm}^{-1}$; MS (EI) m/z: $272\left(\mathrm{M}^{+}\right), 243,195,111,105,77$.

1,1-二环已基-1-苯基甲醇 (4k) ${ }^{[27]}$ : 无色固体, m.p. 76 79 ${ }^{\circ} \mathrm{C}$ (文献值 m.p. $77{ }^{\circ} \mathrm{C}$ ); ${ }^{1} \mathrm{H}$ NMR $(300 \mathrm{MHz}$, $\left.\mathrm{CDCl}_{3}\right) \delta: 7.90(\mathrm{~d}, J=8.3 \mathrm{~Hz}, 1 \mathrm{H}), 7.31(\mathrm{~d}, J=8.3 \mathrm{~Hz}$, $1 \mathrm{H}), 2.58$ (s, 1H), 2.04 0.41 (m, 25H); IR (KBr) v: 3463, 2927, 2851, 1664, 1448, 829, 769, $704 \mathrm{~cm}^{-1}$; MS (EI) $\mathrm{m} / z$ : $272\left(\mathrm{M}^{+}\right), 271,189,171,107,91,83$.

1-(4-甲基苯基)-1,1-二苯基甲醇(4I) ${ }^{[18]}$ : 无色固体, m.p. $70 \sim 72{ }^{\circ} \mathrm{C}$ (文献值 m.p. $65 \sim 66{ }^{\circ} \mathrm{C}$ ); ${ }^{1} \mathrm{H}$ NMR $(300$ $\left.\mathrm{MHz}, \mathrm{CDCl}_{3}\right) \delta: 7.34(\mathrm{~s}, 10 \mathrm{H}), 7.18(\mathrm{~d}, J=4.4 \mathrm{~Hz}, 4 \mathrm{H})$, 2.86 (s, 1H), 2.39 (s, 3H); IR (KBr) v: 3467, 2920, 2361, 1445, 1328, 1153, 814, 758, $699 \mathrm{~cm}^{-1}$; MS (EI) $\mathrm{m} / z: 274$ $\left(\mathrm{M}^{+}\right), 297,183,168,119,105,91,77$.

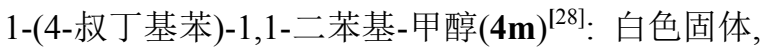
m.p. $80 \sim 82{ }^{\circ} \mathrm{C}$ (文献值 m.p. $80 \sim 81{ }^{\circ} \mathrm{C}$ ); ${ }^{1} \mathrm{H}$ NMR $(300$ $\left.\mathrm{MHz}, \mathrm{CDCl}_{3}\right) \delta: 7.39 \sim 7.31(\mathrm{~m}, 12 \mathrm{H}), 7.28 \sim 7.17(\mathrm{~m}$, 2H), $2.82(\mathrm{~s}, 1 \mathrm{H}), 1.32$ (d, $J=15.6 \mathrm{~Hz}, 10 \mathrm{H})$; IR (KBr) $v$ : 3562, 3463, 2960, 1490, 1446, 1007, 831, 710, $596 \mathrm{~cm}^{-1}$;
MS (EI) $m / z: 316\left(\mathrm{M}^{+}\right), 238,209,183,119,105,91,77$. 1,1-二-(4-甲氧基苯基)-1-(4-叔丁基苯基)甲醇(4n): 无色固体, m.p. $109 \sim 111{ }^{\circ} \mathrm{C} ;{ }^{1} \mathrm{H}$ NMR $(300 \mathrm{MHz}$, $\left.\mathrm{CDCl}_{3}\right) \delta: 7.34(\mathrm{~d}, J=8.5 \mathrm{~Hz}, 2 \mathrm{H}), 7.25 \sim 6.76(\mathrm{~m}, 10 \mathrm{H})$, $3.83(\mathrm{~s}, 6 \mathrm{H}), 2.76(\mathrm{~s}, 1 \mathrm{H}), 1.34(\mathrm{~s}, 9 \mathrm{H}) ;{ }^{13} \mathrm{C}$ NMR $(75$ $\left.\mathrm{MHz}, \mathrm{CDCl}_{3}\right) \delta: 158.6,149.9,144.3,139.7,129.1,127.5$, $124.8,113.1,81.3,55.3,34.5,31.4$; IR (KBr) v: 3503, 2959, 1607, 1508, 1462, 1249, 1177, 1104, 829, $591 \mathrm{~cm}^{-1}$; MS (EI) $m / z: 376\left(\mathrm{M}^{+}\right), 269,243,161,135,118,105,77$; HR-ESI-MS calcd for $\mathrm{C}_{25} \mathrm{H}_{27} \mathrm{O}_{3}:[\mathrm{M}-\mathrm{H}]^{+}$375.1966, found 375.1961 .

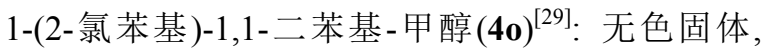
m.p. 89 91 ${ }^{\circ} \mathrm{C}$ (文献值 m.p. 91 92 ${ }^{\circ} \mathrm{C}$ ); ${ }^{1} \mathrm{H}$ NMR $(300$ $\left.\mathrm{MHz}, \mathrm{CDCl}_{3}\right) \delta: 7.43(\mathrm{dd}, J=7.9,1.2 \mathrm{~Hz}, 1 \mathrm{H}), 7.39 \sim 7.24$ $(\mathrm{m}, 10 \mathrm{H}), 7.14(\mathrm{dd}, J=1.27 .8,1 \mathrm{H}), 6.73 \sim 6.78(\mathrm{~m}, 1 \mathrm{H})$, 4.47 (s, 1H, OH); IR (KBr) v: 3565, 3060, 1589, 1441, 1158, 1011, 761, 697, $630 \mathrm{~cm}^{-1}$; MS (EI) $\mathrm{m} / z: 294\left(\mathrm{M}^{+}\right)$, 277, 217, 183, 154, 139, 105, 91, 77;

1,1-二-(2-甲氧基苯基)-1-(4-叔丁基苯基)甲醇 $(4 p)$ 白色固体，m.p. 151 $153{ }^{\circ} \mathrm{C} ;{ }^{1} \mathrm{H}$ NMR $(300 \mathrm{MHz}$, $\left.\mathrm{CDCl}_{3}\right) \delta: 7.37 \sim 6.84(\mathrm{~m}, 13 \mathrm{H}), 3.64 \sim 3.41(\mathrm{~m}, 6 \mathrm{H})$, $1.37 \sim 1.32(\mathrm{~m}, 9 \mathrm{H}) ;{ }^{13} \mathrm{C}$ NMR $\left(75 \mathrm{MHz}, \mathrm{CDCl}_{3}\right) \delta$ : $193.9,157.5,149.4,143.0,134.8,129.6,128.5,127.6$, 124.2, 120.4, 112.5, 81.0, 76.6, 55.7, 34.4, 31.4; IR (KBr) $v: 3550,2951,1934,1591,1104,911,788,634,581$ $\mathrm{cm}^{-1}$; MS (EI) $\mathrm{m} / z: 376\left(\mathrm{M}^{+}\right), 269,243,211,181,165$, 121, 105, 91, 77; HR-ESI-MS calcd for $\mathrm{C}_{24} \mathrm{H}_{25} \mathrm{O}_{3}[\mathrm{M}-$ $\left.\mathrm{CH}_{3}\right]^{+}$361.1798, found 361.1803 .

1,1-二(2-甲氧基苯基)-1-(4-甲基苯)甲醇(4q)：白色 固体, m.p. $124 \sim 127{ }^{\circ} \mathrm{C} ;{ }^{1} \mathrm{H}$ NMR $\left(300 \mathrm{MHz}, \mathrm{CDCl}_{3}\right) \delta$ : $7.35 \sim 7.29(\mathrm{~m}, 1 \mathrm{H}), 7.28 \sim 6.85(\mathrm{~m}, 11 \mathrm{H}), 3.64 \sim 3.51(\mathrm{~m}$, $6 \mathrm{H}), 2.39 \sim 2.35(\mathrm{~m}, 3 \mathrm{H}) ;{ }^{13} \mathrm{C}$ NMR $\left(75 \mathrm{MHz}, \mathrm{CDCl}_{3}\right) \delta$ : $157.5,143.4,136.1,134.6,127.8,120.3,112.4,76.9,55.7$, 21.1; IR (KBr) v: 3532, 2930, 2834, 1905, 1591, 1242, 1052, 918, 748, 630, $582 \mathrm{~cm}^{-1}$; MS (EI) $m / z: 334\left(\mathrm{M}^{+}\right)$, 316, 243, 227, 211, 181, 135, 121, 105, 91, 77; HR-ESIMS calcd for $\mathrm{C}_{21} \mathrm{H}_{18} \mathrm{O}_{3}\left[\mathrm{M}-\mathrm{CH}_{3}\right]^{+} 319.1329$, found 319.1329 .

1,1-二(2-甲基苯基)-1-(4-甲基苯基)甲醇 (4r)：淡黄 色固体, m.p. $105 \sim 107{ }^{\circ} \mathrm{C} ;{ }^{1} \mathrm{H}$ NMR $\left(300 \mathrm{MHz}, \mathrm{CDCl}_{3}\right)$ $\delta: 7.24 \sim 7.02(\mathrm{~m}, 10 \mathrm{H}), 6.79 \sim 6.72(\mathrm{~m}, 2 \mathrm{H}), 2.41(\mathrm{t}, J=$ $5.9 \mathrm{~Hz}, 3 \mathrm{H}), 2.26 \sim 2.15(\mathrm{~m}, 6 \mathrm{H}) ;{ }^{13} \mathrm{C}$ NMR $(75 \mathrm{MHz}$, $\left.\mathrm{CDCl}_{3}\right) \delta: 144.5,143.3,137.7,136.7,132.6,129.1,128.6$, $127.9,127.5,125.0,84.4,22.4,21.1$; IR (KBr) v: 3550, 2924, 1484, 1454, 1011, 819, 778, $638 \mathrm{~cm}^{-1}$; MS (EI) $\mathrm{m} / \mathrm{z}$ : 
$302\left(\mathrm{M}^{+}\right), 284,243,119,104,91$; HR-ESI-MS cacld for $\mathrm{C}_{22} \mathrm{H}_{21} \mathrm{O}[\mathrm{M}-\mathrm{H}]^{+}$301.1598, found 301.1595.

2-甲基-1,1-二苯基-1-丙醇(4s) ${ }^{[11 b]}$ : 白色固体, m.p. 44 46 ${ }^{\circ} \mathrm{C}$ (文献值 m.p. 46 47 ${ }^{\circ} \mathrm{C}$ ); ${ }^{1} \mathrm{H}$ NMR $(300 \mathrm{MHz}$, $\left.\mathrm{CDCl}_{3}\right) \delta: 7.63 \sim 7.00(\mathrm{~m}, 10 \mathrm{H}), 2.93(\mathrm{dt}, J=13.4,6.7 \mathrm{~Hz}$, $1 \mathrm{H}), 2.08(\mathrm{~s}, 1 \mathrm{H}), 0.93(\mathrm{~d}, J=6.7 \mathrm{~Hz}, 6 \mathrm{H})$; IR (KBr) $v$ : 3583, 2970, 1466, 1162, 1008, 757, 701, $632 \mathrm{~cm}^{-1}$; MS (EI) $m / z: 226\left(\mathrm{M}^{+}\right), 183,165,105,77,91$.

辅助材料(Supporting Information) 所合成已知化合 物的 ${ }^{1} \mathrm{H}$ NMR 和 GC/MS 谱图; 新化合物的 ${ }^{1} \mathrm{H} \mathrm{NMR},{ }^{13} \mathrm{C}$ NMR 以及高分辨质谱(HRMS)谱图. 这些材料可以免费 从本刊网站(http://sioc-journal.cn/)上下载.

\section{References}

[1] Corey, E. J.; Cheng, X.-M. The Logic of Chemical Synthesis, John Wiley \& Sons, New York, 1989.

[2] Li, C.-J. Chem. Rev. 2005 105, 3095.

[3] (a) Knochel, P.; Dohle, W.; Gommermann, N.; Kneisel, F. F.; Kopp, F.; Korn, T.; Sapountzis, I. Angew. Chem., Int. Ed. 2003, 42, 4302. (b) Silverman, G. S.; Rakita, P. E. Handbook of Grignard Reagents, CRC Press, New York, 1996.

(c) Richey, H. G. Grignard Reagents: New Developments, John Wiley \& Sons Inc, New York, 2000.

[4] Wakefield, B. J.; Salford, U. O. Organomagnesium Methods in Organic Chemistry, Academic Press, New York, 1995.

[5] (a) Li, C.-J. Tetrahedron 1996, 52, 5643.

(b) Li, C.-J.; C.T.-H. Tetrahedron 1999, 55, 11149.

[6] (a) Kashyap, B.; Phukan, P. Tetrahedron Lett. 2013, 54, 6324.

(b) Augé, J.; Lubin-Germain, N.; Thiaw-Woaye, A. Tetrahedron Lett. 1999, 40, 9245.

(c) Li, C.-J.; Zhang, W.-C. J. Am. Chem. Soc. 1998, 120, 9102.

(d) Keh, C. C. K.; Wei, C.-M.; Li, C.-J. J. Am. Chem. Soc. 2003, $125,4062$.

(e) Hirayama, L. C.; Haddad, T. D.; Oliver, A. G.; Singaram, B. J. Org. Chem. 2012, 77, 4342.

(f) Munoz-Bascon, J.; Sancho-Sanz, I.; Alvarez-Manzaneda, E.; Rosales, A.; Oltra, J. E. Chemistry 2012, 18, 14479.

[7] (a) Oudeyer, S.; Léonel, E.; Paugam, J. P.; Nédélec, J.-Y. Tetrahedron 2014, 70, 919.

(b) Scala, A. D.; Garbacia, S.; Helion, F.; Lannou, M. I.; Namy, J. L. Eur. J. Org. Chem. 2002, 2989.

(c) Chouhan, M.; Sharma, R.; Nair, V. A. Appl. Organomet. Chem. 2011, 25, 470 . (d) Wen, Y.-M.; Chen, G.-F.; Huang, S.-Q.; Tang, Y.; Yang, J.; Zhang, Y.-M. Adv. Synth. Catal. 2016, 358, 947.

[8] (a) Shen, Z.-L.; Loh, T. P. Org. Lett. 2007, 9, 5413.

(b) Lombardo, M.; Morganti, S.; Trombini, C. J. Org. Chem. 2003, 68, 997.

[9] Zhou, F.; Li, C.-J. Nat. Commun. 2014, 5, 4254.

[10] Wen, Y.-M.; Chen, G.-F.; Tang, Y.; Chen, J.; Yang, J.; Zhang, Y.-M. Chin. J. Org. Chem. 2015, 35, 2545 (in Chinese).

(温运明, 陈桂芳, 唐渝, 陈静, 杨骏, 张洣明, 有机化学, 2015, 35,2454 .)

[11] (a) Marie-Isabelle L.; Hélion, F.; Namy, J. L. Tetrahedron Lett. 2002, 43, 8007.

(b) Gao, F.; Deng, X.-J.; Tang, Y.; Tang, J.-P.; Yang, J.; Zhang, Y.-M. Tetrahedron Lett. 2014, 55, 880.

[12] Dishart, K. T.; Levin, R. J. Am. Chem. Soc. 1956, 78, 2268.

[13] Watanabe, S.; Suga, K.; Fujita, T.; Saito, N. Aust. J. Chem. 1977, $30,427$.

[14] Fiandanese, V.; Marchese, G.; Ronzini, L. Tetrahedron Lett. 1983, $24,3677$.

[15] Watanabe, S.; Suga, K.; Yamaguchi, Y. J. Chem. Technol. Biotechnol. 1972, 22, 43.

[16] Li, Y.-C.; Yue, B.; Yang, W.-M.; Xie, Z.-K.; Chen, Q.-L.; Yan, S.-R.; He, H.-Y. Chemistry 2005, 3, 172 (in Chinese). (李应成，岳斌，杨为民，谢在库，陈庆龄，间世润，贺鹤勇，化 学通报, 2005, 3, 172.)

[17] Eisch, J. J.; Liu, W.; Zhu, L.; Rheingold, A. L. Eur. J. Org. Chem. 2015, 7384.

[18] Good, J. A.; Wang, F.; Rath, O.; Kaan, H. Y. K.; Talapatra, S. K.; Podgórski, D.; Kozielski, F. J. Med. Chem 2013, 56, 1878.

[19] Hatano, T.; Kato, T. Tetrahedron 2008, 64, 8368.

[20] Chen, C.; Yang, Y.; Xi, F. J. Polym. Sci., Part A: Polym. Chem. 2001, 39, 430.

[21] Bodendiek, S. B.; Rubinos, C.; Trelles, M. P.; Coleman, N.; Jenkins, D. P.; Wulff, H.; Srinivas, M. Front. Pharmacol. 2012, 3, 106.

[22] Teng, M.-Y.; Liu, Y.; Li, S.-L.; Huang, G.-L.; Jiang, J.-L.; Wang, L.-Y. RSC $A d v$. 2013, 3, 9016.

[23] Horn, M.; Mayr, H. Eur. J. Org. Chem. 2011, 2011, 6470.

[24] Ueura, K.; Miyamura, S.; Satoh, T.; Miura, M. J. Organomet. Chem. 2006, 691, 2821.

[25] Weber, E.; Skobridis, K.; Wierig, A.; Goldberg, I. J. Incl. Phenom. Mol. Recognit. Chem. 1997, 28, 163.

[26] Abarca, B.; Asensio, G.; Ballesteros, R.; Varea, T. J. Org. Chem. 1991, 56, 3224.

[27] Bai, J.-C.; Zhou, W.-K.; Ding, H.-X. J. Organomet. Chem. 1989, $367, \mathrm{C} 9$.

[28] Evans, A. G.; Jones, J.; Osborne, G. Trans. Faraday Soc. 1954, 50, 16.

[29] Wang, F.; Good, J. A.; Rath, O.; Kaan, H. Y. K.; Sutcliffe, O. B.; Mackay, S. P.; Kozielski, F. J. Med. Chem. 2012, 55, 1511. 